\title{
Processing of semantic and grammatical gender in Spanish speakers with aphasia
}

\author{
G. Piazza ${ }^{\mathrm{a}, \mathrm{b} *}$, M. Calabria ${ }^{\mathrm{c}}$, C. Semenza ${ }^{\mathrm{d}}$, C. Poletto ${ }^{\mathrm{b}, \mathrm{e}}$
}

${ }^{a}$ Basque Center on Cognition, Brain and Language (BCBL), Donostia-San Sebastián, Spain;

${ }^{b}$ Department of Linguistic and Literary Studies (DiSLL), University of Padova, Padova, Italy;

${ }^{c}$ Faculty of Health Sciences, Universitat Oberta de Catalunya (UOC), Barcelona, Spain;

${ }^{d}$ Padova Neuroscience Center (PNC), University of Padova, Padova, Italy;

${ }^{e}$ Institute of Romance Languages and Literature, Goethe University Frankfurt, Frankfurt am Main, Germany.

*corresponding author: g.piazza@bcbl.eu

Author Note

This is a preprint of the paper published in Aphasiology. Please, find the published version at https://www.tandfonline.com/doi/full/10.1080/02687038.2021.1924355 


\section{Processing of semantic and grammatical gender in Spanish speakers with aphasia}

Abstract

Background. Previous studies have argued that two types of linguistic gender exist: grammatical gender, which is arbitrarily assigned to nouns, and semantic gender, which depends on the gender of the referent.

Aim. We explore the hypothesis that these two types of gender entail distinct cognitive processes by investigating the performance of people with aphasia at the level of sentence comprehension.

Methods and Procedure. Eleven people with aphasia and a control group of 13 agematched healthy participants took part in a constrained completion choice task. The participants had to complete sentences in a way that made the last word gender congruent. The subjects of the sentences had either Semantic gender ("enfermera", nurse; indicating the sex of the referent), Grammatical gender ("silla", chair), or Opaque-Grammatical gender ("tomate", tomato).

Results. People with aphasia performed more poorly in all gender conditions than healthy controls. They also were less accurate in both the Grammatical and Opaque-Grammatical conditions than in the Semantic gender condition.

Conclusion. We propose that semantic and grammatical gender entail two levels of gender processing and that semantic gender is processed faster because it provides more salient information. 
Keywords: aphasia, agreement, semantic gender, grammatical gender, sentence comprehension.

\section{Introduction}

Gender processing is a critical part of both lexical and syntactic processing in many languages. Transparent languages convey explicit grammatical gender information in the final morpheme of nouns or through a noun affix. For instance, Spanish is considered (morphologically) transparent because the (transparent) final morpheme of nouns provides gender information: -a for words with feminine grammatical gender (e.g. "casa”, house.FEM), -o for masculine words (e.g. "casco", helmet.MASC). However, in some cases, the final morpheme -e (opaque) does not permit gender identification because it is used for both feminine and masculine nouns. Furthermore, there are two different types of gender: grammatical gender is arbitrarily assigned to nouns, while semantic gender is dictated by the gender of the referent ${ }^{1}$. In Spanish, both types of gender follow the same morphemic rules and use the same features. For instance, changing the ending (-o/-a) creates a noun of the opposite gender with some, but not all, animal names: "mono" (male monkey) or "mona" (female monkey). Semantic gender is presumed to be strictly bound to the semantic meaning of nouns and for this reason is hypothesized to involve a different retrieval path than grammatical gender (Vigliocco and Franck, 1999).

In the present study, we investigate the hypothesis that processing grammatical gender and semantic gender entail two levels of comprehension processes. We explore the assumption that semantic gender carries salient semantic information - making it easier to process agreement and access meaning - by comparing the performance of people with aphasia (henceforth PWA) with healthy controls. In fact, studies on various populations of patients have highlighted that 
gender can be preserved, lost, or difficult to retrieve in word recognition and sentence comprehension (e.g., Manenti et al., 2009), suggesting possible dissociations between these linguistic processes.

\subsection{Semantic vs grammatical gender}

The difference between semantic and grammatical gender is not merely a difference between animate and inanimate nouns. Semantic gender is supplementary semantic information about the gender of the noun referent that is reflected in the noun's superficial morphology. For instance, it is distinct from grammatical gender in words like "silla" (chair.FEM), and "pájaro" (bird.MASC), where the morphology does not convey any information about the sex of the referents; in fact, a chair is an object, while the word "pájaro" does not have a feminine counterpart and is a general term that refers to all birds, both male and female. It is worth underscoring that the gender or sex of an animate referent does not in itself constitute semantic gender if it does not coincide with the morphological expression of gender. For this reason, "pájaro", which does not have a feminine counterpart, and "tigre" (tiger.MASC), which ends with a morpheme that could be either masculine or feminine, do not have semantic gender.

Comprehension and information retrieval mechanisms operate differently when nouns are encountered in the context of sentences than they do in bare noun recognition. Accordingly, while agreement is optional in word pairs, it is always active and involves a constant process for selection of correct agreement candidates in sentences (see Roelofs et al., 1998; Cacciari and Cubelli, 2003; Corbett, 2006). For this reason, our experimental design used an enriched sentence comprehension context, which ensured gender was activated as participants accessed word meaning and computed agreement at a distance. 
There are two main accounts that aim to explain how semantic gender influences sentence comprehension. The first account is known as the minimal input hypothesis and derives from theories that claim semantic features have minimal impact on syntactic processing (Bock and Levelt, 1994; Kempen and Hoenkamp, 1987; Levelt, 1989). The minimal input hypothesis claims that grammatical gender is a purely syntactic feature, which cannot be influenced by conceptual agreement ${ }^{1}$ (Chomsky, 1965, 1981); "the committee have decided" is an example of conceptual agreement. In this sentence, the subject-verb agreement is not correct from a grammatical point of view, but it is conceptually acceptable because the meaning is plural. When possible, syntactic agreement is preferred over conceptual agreement; children, for example, autonomously create an agreement system between constituents before they learn rules and irregularities. In this first account, there cannot be different levels (grammatical and semantic) for processing gender, because gender is grammatical and therefore is only processed at the syntactic level. The second account is called the maximal input hypothesis and claims that semantic meaning and structures have some influence over agreement and syntactic operations (Barlow, 1993; MacDonald et al., 1994; Pollard and Sag, 1994; Vigliocco, et al., 1996; Vigliocco and Franck, 1999).

Since many linguistic phenomena demonstrate a dichotomy between semantic and grammatical gender, Vigliocco and Franck (1999) postulate that they are, to some extent, processed differently. According to this view, conceptual structures that carry semantic information influence grammatical functions and syntactic processes (such as agreement). Additional support for this hypothesis comes from work on argument hierarchy and featuresharing, which shows that agreement is driven by semantic as well as syntactic relations. This has led to the assumption that gender has a double nature (Vigliocco and Franck, 1999), which allows us to process semantic gender differently from grammatical gender (cf. Van Dyke, 2007; 
for a different view see Bates et al., 1995). Linguistic elements create dependencies, even at long distances, which comprehension processes resolve via cue-based retrieval mechanisms (Lewis et al, 2006; Van Dyke and McElree, 2006). If a competing item shares some features with the target, that can interfere with the retrieval process and induce errors (Parker et al., 2017). Conversely, semantic cues would also explain why semantic gender nouns were produced more accurately than grammatical gender nouns in Vigliocco and Franck's experiment (1999).

In the study by Vigliocco and Franck (1999), participants were first presented with adjectives in both their masculine and feminine forms, then a preamble sentence. The gender type of the subject (head noun of the sentence) had either semantic or grammatical gender. The participants had to repeat the whole sentence, adding on the correct (gender-matched) adjective at the end. The researchers found that agreement errors were more frequent when the subjects had grammatical gender. They argued that their manipulation led participants to distinguish between gender that carries both syntactic information and semantic properties derived from the gender of the referent (semantic gender) from purely grammatical gender with bare syntactic features (grammatical gender). Although we still lack evidence for this division of labour in sentence comprehension processing, such results strongly suggest that at least in speech production - some kind of internal gender distinction is made, and that different processes underpin the assignment of semantic and grammatical gender (see also Vigliocco et al, 1995; for similar results in comprehension, see Franzon et al., 2013).

Vigliocco and Franck's (1999) experiment also explored whether semantic gender is actually assigned on the basis of animacy, given that all nouns endowed with semantic gender are also animate nouns. They compared animate nouns with transparent gender morphology, in which gender marking was the same for both sexes (as in the "pájaro" example mentioned above) and inanimate nouns. This allowed them to manipulate animacy separately, isolating 
potentially confounding factors. In this experiment, they did not find any difference between animate nouns with fixed gender - a single final morpheme (such as "pájaro") - and inanimate nouns, indicating that semantic gender is not assigned on the base of animacy. Moreover, Vigliocco and Franck (1999) investigated whether animate nouns with fixed grammatical gender bear semantic gender but did not find an effect comparable to that seen for proper semantic gender nouns. These results indicate that semantic gender is processed differently than fixed gender.

This dissociation between semantic and grammatical gender has been demonstrated by a number of studies using different types of paradigms and techniques. Schiller et al. (2003) found that the frontal ERP N200 component was larger for nouns marked with semantic gender than for animate nouns with opaque endings. Similarly, Franzon et al.'s (2013) preliminary study on two people with aphasia confirmed Vigliocco and Franck's (1999) results, finding they had higher accuracy for semantic gender in noun-adjective pairs. In a more recent study, Caffarra et al. (2016) reported an effect of semantic gender on bare words in Spanish-Basque bilinguals. In this experiment, nouns were presented on a screen and the participants had to decide which gender they belonged to. The authors manipulated the nouns in such a way that half of the stimuli had opaque endings (i.e., the final -e in Spanish), which did not provide information on the gender of the nouns, while the other half had transparent endings (i.e. final -a/-o) expressing feminine/masculine gender. Within the transparent category, half of the nouns had semantic gender and half had only grammatical gender. They observed facilitation in terms of RTs and accuracy for nouns bearing semantic gender. This finding suggests that semantic gender helps with the categorisation of bare nouns by means of explicit gender knowledge. However, to date, no study has explored how people with aphasia (PWA) compute semantic gender in comprehension and whether it facilitates agreement processing when no declarative 
knowledge is required (i.e., when deciding whether a noun is masculine or feminine vs using gender to match a noun and its adjective).

In sum, research has not provided conclusive evidence for the existence of two types of gender processes nor found evidence for it in comprehension tasks. More importantly, it is not clear whether semantic gender facilitates processing, or simply differs from grammatical gender. The present study intends to contribute to clarifying these issues using a cognitive neuropsychology method.

\subsection{Aphasia and Gender Processing}

Research has found evidence for and against the maintenance of gender knowledge in PWA. Some research has demonstrated that gender knowledge is maintained in people with aphasia. For instance, although Bates et al. (2001) tested both Broca's and Wernicke PWA and failed to observe gender priming effects, they found that PWA were able to correctly use gender. In contrast, Akhutina et al. (2001) found that PWA, with various types of aphasia, had comparable difficulties with recognizing grammatical gender during a grammaticality judgement task. The apparent discrepancy between these results can be explained if we assume that two types of gender knowledge exist: one procedural and the other declarative or metalinguistic (Scarnà and Ellis, 2002). The former is unconscious syntactic gender knowledge that allows for automatic computation of agreement between constituents without the need for explicit recall of gender. The latter is the metalinguistic ability to explicitly indicate the gender of an element without putting any syntactic operations into play (Goodglass, 2000, see also Miceli et al, 2002). Scarnà and Ellis (2002) made this assumption after they found that bilingual PWA had problems categorizing Italian nouns with explicit grammatical gender, but were nevertheless able to modify adjectives to ensure agreement 
with a Noun Phrase (NP). It appears that, in PWA, grammatical gender remains intact when retrieved procedurally and unconsciously employed for agreement but is impaired when consciously recalled.

Despite the evidence reported above, some research on PWA has shown varying results for procedural knowledge. For instance, Mondini et al. (1999) tested an Italian with aphasia on article-noun production and described serious deficits in producing gender agreement even for nouns bearing gender information, (e.g., "il madre", the.MASC mother). It is worth noting that, because of the opaque final -e, cases like "madre" do not allow for direct recall of gender information ("padre", father ends with the same final -e), so it may not be possible to observe the semantic gender facilitation, as Vigliocco and Franck (1999) also pointed out. Friedmann and Biran (2003) studied picture naming, which does not require declarative gender knowledge since gender is only used to produce agreement (by assigning the correct gender morpheme), in Hebrew-speaking PWA. Their participants did not preserve grammatical gender. The authors suggest that this result could reflect the special features of Hebrew which allows bare nouns - these nouns do not need a determiner to be linguistically legal. Consistent with what we previously reported in Cacciari and Cubelli (2003), grammatical gender might only be accessed if agreement is activated (see Roefols et al., 1998). Again, for this reason we decided to use subject-adjective pairs in sentence comprehension, to make sure that agreement comes into play. Moreover, congruent with evidence on explicit and procedural gender knowledge distinction (Scarnà and Ellis, 2002), we expected PWA to be able to unconsciously employ gender information in our experiment, where adjective and noun gender is procedurally matched, without any need for categorisation.

In sum, evidence from PWA is neither clear-cut nor definitive. In addition, to the best of our knowledge, semantic gender comprehension has never been systematically investigated 
in aphasic populations (see Semenza and Luzzatti, 2019). It is not known how semantic gender is processed by PWA, that is, whether impairments in gender-explicit knowledge may influence their performance in comprehension, or whether - as we assume - additional semantic information on the sex of the referent will help them complete the task, as we expect to find in control participants. As demonstrated by prior research, cognitive processes in impaired and healthy populations diverge substantially; by pinpointing specific deficits, we can learn more about such processes.

\section{The Present Study}

This study aimed to verify whether gender is processed differently during sentence comprehension when its source (e.g., sex) matches morphology, and whether explicit knowledge impairments differently influence the performance of PWA when restoring grammatical and semantic gender. In a constrained completion choice task, participants read a sentence and then saw - in isolation - the masculine and feminine forms of an adjective; they had to decide which form matched the subject of the previous sentence. We employed three conditions: Grammatical gender, Semantic gender (which shares the morphological transparency of the first condition), and Opaque-Grammatical gender, a condition inserted to prevent participants from basing their strategy solely on morphology and to check whether any of the observed effects could be driven by morphology.

According to the maximal input hypothesis (Vigliocco \& Franck, 1999), which proposes that semantic processing exerts an influence on grammatical processing, we expected the following results:

Healthy controls would respond faster when checking agreement between adjectives and subjects bearing semantic compared to grammatical gender. As the task is easy for healthy 
individuals, we did not expect gender conditions to influence their accuracy. In fact, their performance might show reduced differences between conditions and hide the effect derived from processing semantic gender.

We expected patients to be less accurate and slower in all conditions than healthy controls. We were interested to understand if this impairment would vary with gender type; we expected PWA to be faster and more accurate in the Semantic condition. Specifically, if patients performed better in the Semantic than the other conditions, this would confirm the maximal input hypothesis. Furthermore, unlike healthy controls, they might have issues in computing agreement for grammatical and opaque-grammatical gender, which would widen the gap between these two grammatical conditions and the Semantic condition. Alternatively, if patients perform similarly in all gender conditions, we would have to conclude that gender types are not dissociated, the maximal input hypothesis could not explain our results, and the two gender types do not follow two distinct processing paths.

\section{Method}

\subsection{Participants}

A total of 11 Spanish-speaking people (7 Spanish-Catalan bilinguals) with aphasia were recruited from the Speech Therapy Unit of the San Pau i la Santa Creu Hospital in Barcelona (mean age 50 years \pm 9.63 , mean education 13.7 years \pm 3.6 , aphasia severity range from mild to moderate). Ten PWA had suffered a stroke and one had had cerebral cancer. All the PWA had a brain lesion in the left hemisphere, exhibited adequate hearing, demonstrated stable health status, and were in the chronic stage of language disorders (more than one year after the injury). From the total number of PWA, two were discarded from the analysis due to their high error rate (around 60\%) leaving a final cohort of 9 PWA. 
A control group, comprising 13 participants matched for age and education with the PWA group, also completed both tasks. Eleven of these participants were native Catalan-Spanish speakers, while two were Spanish speakers with some knowledge of Catalan (passive bilinguals; mean age $50 \pm 4.6$, mean education 14.1 years, \pm 2.3$){ }^{2}$

Before starting the experimental procedure, both patients and controls signed an informed consent form approved by the 'Parc de Salut MAR' Research Ethics Committee.

Language assessment. To define the type and degree of language impairment in PWA participants, a Spanish version of the Western Aphasia Battery test (WAB, Kertesz, 1982; Kertesz \& Pascual-Leone, 2000) was administered by a neuropsychologist with expertise in aphasia. The WAB allows for the evaluation of the main clinical aspects of language: function, content, fluency, auditory (sentence) comprehension, repetition, naming, reading, writing, and calculation.

According to the WAB assessment, and according to the subtest scores (see Table 1 for individual subtest scores), only one PWA exhibited NonFluent aphasia along with scores compatible with Broca's aphasia (Pt. 5); the rest exhibited Fluent aphasia. The subtest scores of three PWA were compatible with Anomic aphasia, two with Wernicke's aphasia, and two with Conduction aphasia. One PWA was not classified (Pt.9).

The degree of language impairment ranged from mild to moderate (WAB AQs of 54.1 to 89.2 out of 100$)$ and the mean values for each subtest were: 13.12/20 $( \pm 4.02)$ for Fluency, 8.23/10 $( \pm 1.40)$ for Comprehension, $6.83 / 10( \pm 1.9)$ for Repetition, and 7.30/10 $( \pm 1.43)$ for Naming.

\section{INSERT TABLE 1 ABOUT HERE}

\footnotetext{
2 See the published version for more details about participants' language profile.
} 


\subsection{Material}

A set of 78 sentences, comprising 26 sentences for each of the three experimental conditions (Gender Type), was selected (see Appendix A for examples). Sentence subjects (or controllers, see Corbett, 1991, 2006) across the three gender conditions differed as follows:

a) Subjects with Semantic gender were nouns that had semantic gender reflecting the sex of the referent (nouns referring to humans, animals, or occupations) and transparent endings (a/-o; e.g., "esposo", spouse.MASC).

b) Subjects with Opaque-Grammatical (henceforth, Opaque) gender were inanimate nouns with grammatical gender and opaque endings (-e ; e.g., "puente”, bridge.MASC).

c) Subjects with Transparent-Grammatical (henceforth, Grammatical) gender were inanimate nouns with grammatical gender and transparent endings (-a/-o; e.g., "cuchara", spoon.FEM).

Adjectives available for sentence completion were all phonologically transparent with -a or -o endings that clearly indicated feminine or masculine gender. The sentences (e.g., "El esposo en la iglesia está", the spouse in the church is...) were formed with a subject that was a full noun-phrase (NP), including an article and a noun, followed by a distractor that always had the opposite gender as the subject ("en la iglesia", in the church) and the third-person singular of the verbs ser/estar (to be; hence, es or está). The final part of the sentence was the adjective, which needed to agree with the subject. All adjectives had transparent endings (-a/-o) with relevant features balanced across conditions (see below); all options provided plausible sentence completions (e.g., "emocionado", excited). We introduced distractors with the opposite gender from subjects to induce a higher error rate, since research has shown that target 
retrieval is slower when other items share some of its features (Van Dyke and McElree, 2006; see also Parker et al., 2017).

We checked the values of the psycholinguistic variables of interest using Busca-Palabras (B-Pal) software (Davis \& Perea, 2006); B-Pal is a free software program that brings together previous databases on various psycholinguistic properties (also subjective ratings) of approximately 31,500 Spanish words. The final number results from the authors' selection of words from the various databases and the Real Academia Española (RAE) dictionary. We used B-Pal scores to balance the subjects of the sentences for average log frequency means, which did not significantly vary across conditions: $1.16(\mathrm{SD}=0.53)$ for the Grammatical gender condition, $1.15(\mathrm{SD}=0.6)$ for the Semantic gender condition, and 1.11 $(\mathrm{SD}=0.66)$ for the Opaque gender condition. We did the same for the adjectives and distractors. Adjective average $\log$ frequency did not significantly differ across conditions: $1.25(\mathrm{SD}=0.57)$ for the grammatical, $1.14(\mathrm{SD}=0.5)$ for the semantic, and $1.15(\mathrm{SD}=0.61)$ for the opaque gender condition. The average frequency of distractors was, respectively, $1.37(\mathrm{SD}=0.56), 1.6(\mathrm{SD}=$ $0.66)$, and $1.59(\mathrm{SD}=0.52)$ for the three conditions.

Imageability was also balanced and did not significantly differ across conditions for either adjectives or distractors. The average imageability score for adjectives was $4.01(\mathrm{SD}=0.67)$ for the Grammatical condition, $4.15(\mathrm{SD}=0.51)$ for the Semantic condition, and $4.27(\mathrm{SD}=$ 0.62) for the Opaque condition. The average imageability score of the distractors was 5.59 (SD $=0.91), 5.55(\mathrm{SD}=0.74)$, and $5.83(\mathrm{SD}=0.51)$, respectively.

Since objects do not have semantic gender, it was by definition impossible to balance the animacy scores of subjects across conditions. To calculate the statistics, we simply assigned 0,5 to inanimate and 0,5 to animate nouns. Subjects with Semantic gender had a significantly higher animacy score than the other conditions $(\mathrm{p}<0.05)$, while Opaque and Grammatical gender did not differ. This is partially interesting in itself since, as already mentioned, Vigliocco 
and Franck (1999) tested whether animacy carries the full effect of semantic gender. They found instead that the Semantic gender effect distinguished between conditions with the same level of animacy. Furthermore, they found no effect of animacy per se, indicating the results from the gender type manipulation could not be attributed to animacy (see Vigliocco \& Franck, 1999, for further discussion).

We created 4 different lists to avoid order effects or effects due to random noun-adjective associations. The sentence-adjective pairs were not randomized in order to avoid nonsense sentence-adjective pairs but were varied across the 4 lists. We picked one list of stimuli for every participant in consecutive order. The order of stimulus presentation was randomized.

\subsection{Procedure}

The experimental software used for the administration of all tasks was DMDX (Forster \& Forster, 2003). The experiment was displayed on a 15-inch Toshiba screen with the participant seated in front of the screen at a distance of approximately $40 \mathrm{~cm}$. A fixation cross was presented at the centre of the screen for $700 \mathrm{~ms}$, then a sentence appeared for $3500 \mathrm{~ms}$. The sentences, for example, "El esposo en la iglesia está" (The spouse in the church is) appeared in the mid-upper area of the monitor. In all stimuli, the sentential subject was followed by a filler phrase (e.g., “en la iglesia") and the verb es or está. ${ }^{3}$. The filler phrase (also called the distractor) was included to increase demands on cognitive control. After the initial sentence presentation (3500ms), two options for the final adjective were presented below (in the mid-lower area of the screen) to the left and right, for example, the masculine ("emocionado ", excited) on one side and the feminine ("emocionada", excited) on the other. Right and left positions were

\footnotetext{
${ }^{3}$ In Spanish the verb to be can be expressed using two forms, deriving from different roots, depending on the context.
} 
balanced across participants and trials. The sentence remained on the screen for up to 10 seconds or until a response was provided. The sentences were black (RGB 0,0,0) in size 16 (Font Corbel) presented in the centre of the screen with a grey background (RGB 192,192,192). The adjectives were written in all caps and presented in the centre mid-lower area of the screen. Using a standard keyboard (see Damian, 2010, for a discussion on its experimental use), participants had to choose between the two options in order to complete the sentence according to the gender of the subject: pressing ' $Z$ ' for the left-sided and ' $M$ ' for the right-sided adjective. We substituted keys ' $\mathrm{Z}$ ' and ' $\mathrm{C}$ ' for PWA who could only use their left hand. We counterbalanced the side of the correct answer for all participants in order to reduce any bias on RTs due to hemiplegia. Moreover, participants were asked to initiate responses starting from a neutral position on the keyboard.

\subsection{Statistical analysis}

Repeated-measure ANOVAs were performed for the analysis of the experimental data, including within-subject and Group factors (PWA vs. control group). The analyses were conducted separately on RTs and accuracy. All missed and overtime responses (exceeding timeout), software malfunctions, and inaccurate responses were classified as errors and discarded from the analysis. In addition, we removed all responses exceeding 2 standard deviations above or below the individual RT mean.

Given that Broca's aphasia (NonFluent aphasia) can entail issues with grammar, and therefore also issues with processing grammatical gender, we conducted an analysis on the PWA excluding the participant with Broca's aphasia. In addition, we took into consideration the behaviour of Pt.9 who was not classified, and we checked whether their behavioural pattern was in line with that of participants with Fluent aphasia. Since the pattern of results and the 
analysis without the participant with Broca's aphasia did not change the overall results, here we report only the main analysis conducted. However, individual data from the PWA were analysed and are presented in Figures 2 and 4.

\section{Results}

Given the relatively small sample size, all data were also analysed using non-parametric analyses. As the pattern of results did not differ, here we report only results from the parametric statistics. When significant effects were found in the main analysis, we employed Bonferroni correction for multiple comparisons in post-hoc analyses.

Repeated-measure ANOVAs were performed, including Gender Type (Grammatical vs. Opaque vs. Semantic) as within-subject factors and Group as the between-subjects factor.

Reaction times $(R T S)$. The main effect of Gender Type was significant $[\mathrm{F}(2,40)=6.256$, $\left.\mathrm{p}=0.004, \eta p^{2}=0.238\right]$. The post-hoc analysis showed that participants were faster in the Semantic $(2006 \mathrm{~ms})$ than the Grammatical $(2063 \mathrm{~ms}, \mathrm{p}=0.006)$ and Opaque $(2043 \mathrm{~ms}, \mathrm{p}=0.01)$ conditions (Fig. 2). The interaction between Gender Type and Group did not reach significance $[\mathrm{F}(2,40)=2.590, \mathrm{p}=0.08]$, while the Group effect did $\left[\mathrm{F}(1,20)=16.546, \mathrm{p}=0.001, \eta \mathrm{p}^{2}=\right.$ 0.453], revealing that PWA overall were slower (2943ms) than the control group (1084ms).

In order to control for bilingualism as a possible confounding factor, we conducted a further analysis including only bilingual participants from both groups. This revealed that the Gender Type effect was still significant $\left[\mathrm{F}(2,34)=5.090, \mathrm{p}=0.01, \eta \mathrm{p}^{2}=0.230\right]$, as was a Gender Type*Group interaction $\left[\mathrm{F}(2,34)=3.287, \mathrm{p}=0.050, \eta \mathrm{p}^{2}=0.162\right]$, possibly suggesting that the two groups carried out the task differently. To explain this interaction, we performed further analyses by comparing the magnitude of the effects of the conditions in the two groups of participants. The magnitude of the effects was calculated as follows: for the Semantic condition, we calculated the difference in RTs between the Semantic and the Grammatical 
conditions, divided by the RTs of the Semantic condition and multiplied by 100 (as in Calabria et al., 2012). For the Opaque condition, we calculated the difference in RTs between the Opaque and the Grammatical conditions, divided by the RTs of the Grammatical condition and multiplied by 100 . Despite the interaction, the magnitude of the Semantic gender effect (Semantic-Grammatical) did not differ between the two groups $[F(1,17)=0.009, p=0.92]$. The interaction effect was more likely driven by the Opaque condition. In the control group, the Opaque condition resulted in slower RTs (1076ms) than the Grammatical (1044ms) and the Semantic $(989 \mathrm{~ms})$ conditions, whereas in the PWA group the Grammatical condition was the slowest $(3233 \mathrm{~ms})$, followed by the Opaque $(3039 \mathrm{~ms})$ and Semantic $(2986 \mathrm{~ms})$ conditions. Nevertheless, the magnitude of the Opaque-Grammatical effect did not differ for the two groups $[\mathrm{F}(1,17)=1.133, \mathrm{p}=0.3]$

Individual level analysis. Given that variability of performance in the PWA group might reflect different types of aphasia, we ran an individual level analysis to assess whether there was a substantial difference in the magnitude of the effect in PWA compared to the control group. We used the magnitude of the Semantic gender effect (vs. the Grammatical condition) - as explained above - and applied the modified t-test described by Crawford and Howell (1998) for independent samples. The $\mathrm{t}$ values were calculated as follows:

$$
t=\frac{X_{1}-X_{2}}{s_{2} \sqrt{\frac{N_{2}-1}{N_{2}}}}
$$

where $\mathrm{X}_{1}$ represents individual performance, $\mathrm{X}_{2}$ represents the mean of the control group, $\mathrm{S}_{2}$ is the standard deviation of the control group, and $\mathrm{N}_{2}$ is the sample size of the control group. The analysis showed that 2 PWA had proportionally shorter RTs in the Semantic condition (vs the Grammatical condition) in comparison to the mean score of the control group, Pt. $4-13,7 \%$ 
$[t=2.5, \mathrm{df}=12, \mathrm{p}=0.03]$, Pt.5 $-13,3 \%[\mathrm{t}=2.45, \mathrm{df}=12, \mathrm{p}=0.03]$, suggesting that this group benefitted most from the Semantic condition. In contrast, the Semantic condition slowed down Pt.1's responses, as shown by the proportional difference between the Semantic and Grammatical conditions $[\mathrm{t}=2.25, \mathrm{df}=12, \mathrm{p}=0.04]$.

Fig. 1. RTs broken down by Gender Type and participant group (control group and PWA). The error bars indicate SE. The effect of Group is not reported, even though it is significant.

Constrained Completion Choice: RTs

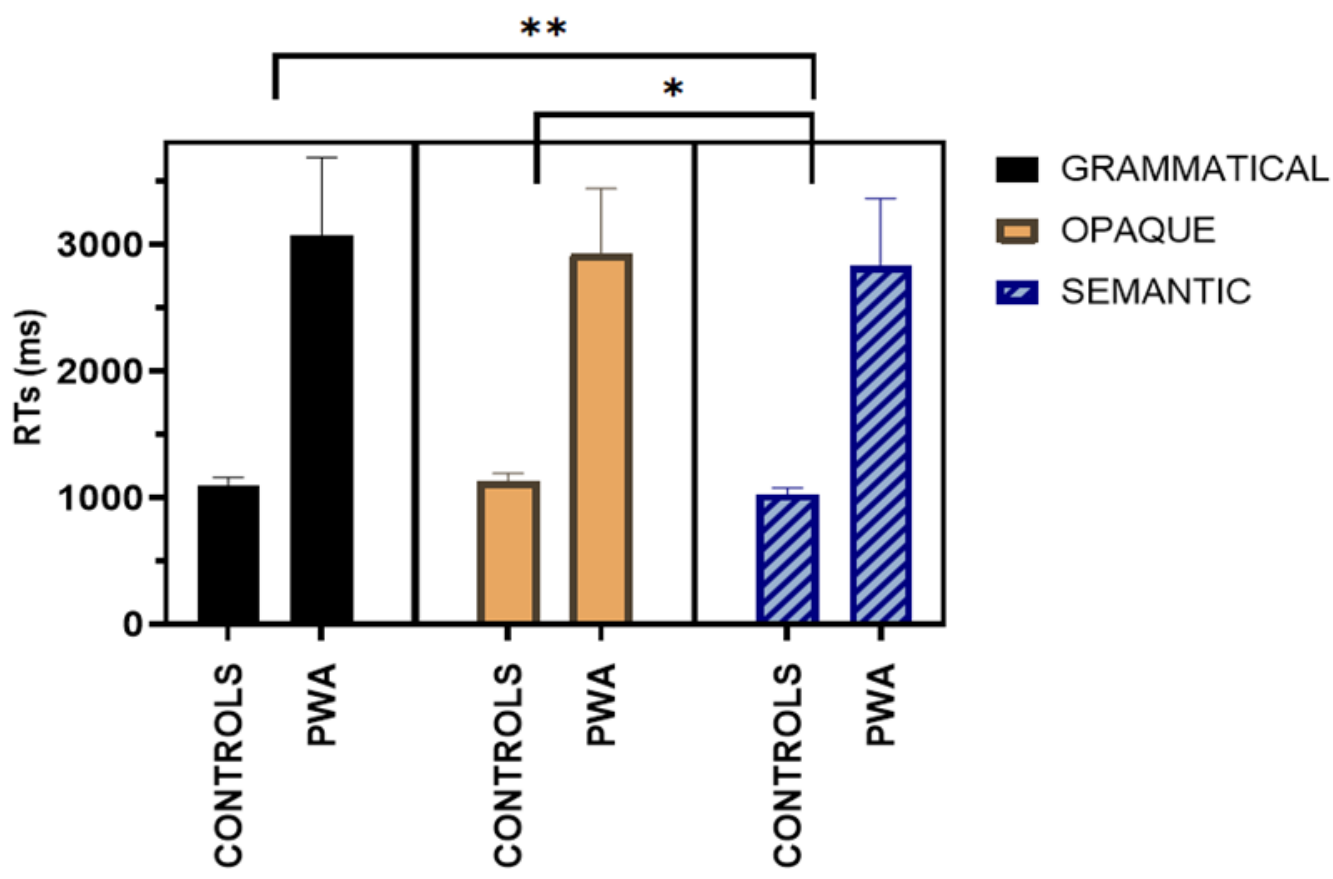


Fig. 2. Magnitude of the Semantic gender effect based on RT differences between the Semantic condition and the Grammatical condition. Individual PWA scores (stars indicate where individual performance significantly differed from the controls). More negative values indicate (proportionally) faster responses in the Semantic condition.

\section{Semantic gender effect (RTs) Individual PWA scores}

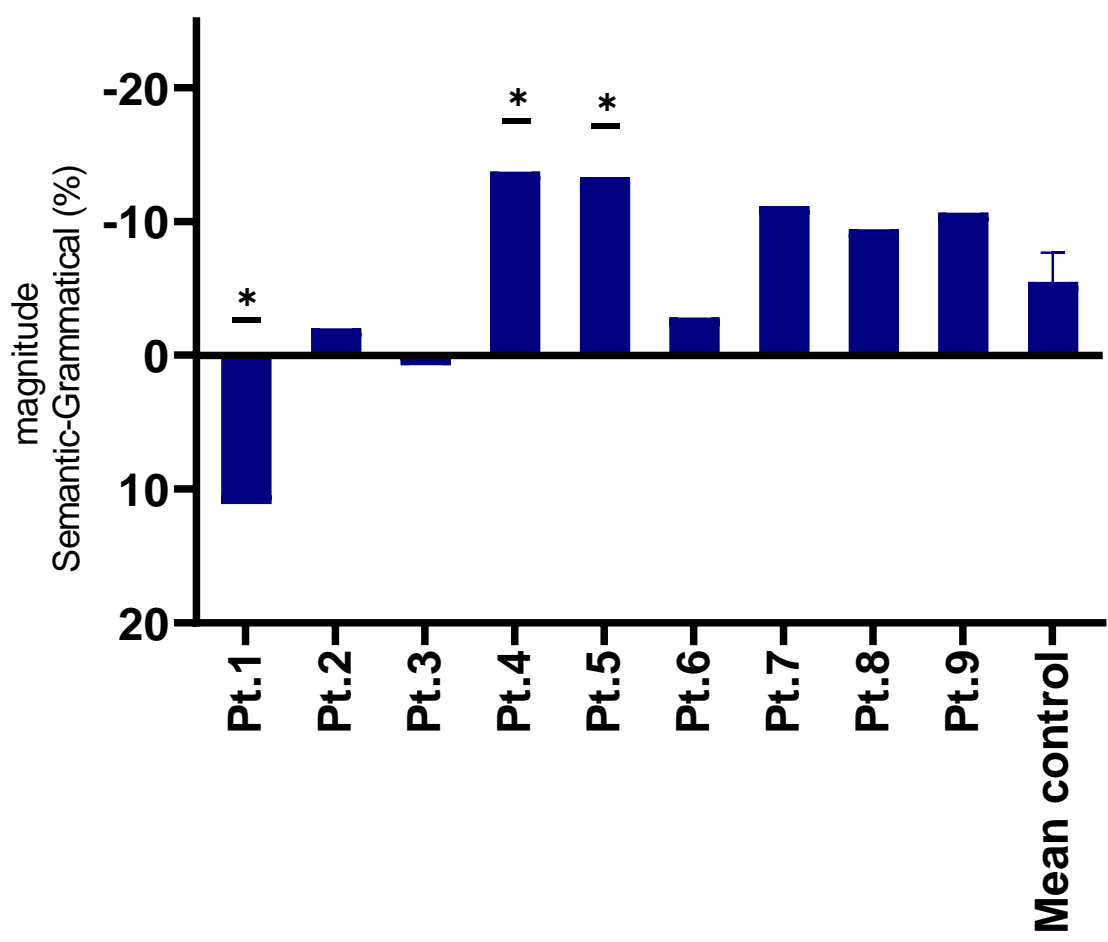

Accuracy. The main effect of Group was significant $\left[\mathrm{F}(1,20)=33.893, \mathrm{p}<0.001, \eta \mathrm{p}^{2}=\right.$ 0.629] suggesting that PWA (74.92\%) performed more poorly than controls $(98.32 \%)$. The main effect of Gender Type was significant $\left[\mathrm{F}(2,40)=6.950, \mathrm{p}=0.003, \eta p^{2}=0.258\right]$ and the post-hoc analysis showed that performance on Semantic $(92,31 \%)$ was significantly more accurate than Opaque $(85,67 \%, \mathrm{p}=0.01)$, and marginally better than Grammatical $(88,29 \%, \mathrm{p}$ $=0.07)$ gender, while the difference between the latter conditions was not significant $(p=0.14)$ (Fig. 3). Also, the interaction between Gender Type*Group was significant $[F(2,40)=4.272$, $\left.\mathrm{p}=0.02, \eta \mathrm{p}^{2}=0.176\right]$. To explore this interaction, we conducted further post-hoc analyses and 
found that the magnitude of the Semantic-Grammatical effect (Semantic gender effect) was significantly greater in PWA [F $\left(1,20=4.542, \mathrm{p}=0.04, \eta \mathrm{p}^{2}=0.185\right]$, whereas the OpaqueGrammatical effect was not significantly different between groups $[F(1,20)=0.905, p=0.3]$ indicating that they had more difficulty processing agreement in the Opaque and Grammatical conditions than controls, whereas the way in which they processed semantic gender was more similar to the control group, therefore more preserved in the Semantic condition. The Analysis on bilingual participants confirmed the above reported results: the main effect of Gender Type was significant $\left[\mathrm{F}(2,34)=4.940, \mathrm{p}=0.01, \eta \mathrm{p}^{2}=0.225\right]$, and there was a Gender Type*Group interaction $\left[F(2,34)=4.097, p=0.02, \eta p^{2}=0.194\right]$. Post-hoc analysis indicates that the Semantic condition significantly differed from both the Grammatical $(p=0.009)$ and Opaque $(p=0.04)$ conditions, which did not differ from each other $(p=1)$.

Individual level analysis. We ran an individual level analysis on the magnitude of the Semantic gender effect (Semantic-Grammatical) found in PWA to compare each individual's performance to the control group mean. The analysis showed that 6 PWA had a larger magnitude Semantic gender effect compared to the control group mean (Fig. 4): Pt.1 +21\% [t $=5.75, \mathrm{df}=12, \mathrm{p}<0.0001]$, Pt. $2+60 \%[\mathrm{t}=16.75, \mathrm{df}=12, \mathrm{p}<0.0001]$, Pt.7 $+13 \%[\mathrm{t}=3.57$, df $=12, p=0.004]$, Pt. $8+21 \%[t=5.75, d f=12, p<0.0001]$, Pt. $9+18 \%[t=4.94, d f=12, p$ $=0.0003]$. By contrast, Pt.5 showed the reverse pattern of effects with a significantly lower magnitude Semantic gender effect $[t=6.15, \mathrm{df}=12, \mathrm{p}<0.0001]$. Pt.3 and Pt.4, whose magnitude did not significantly differ from the controls', nevertheless showed clear directionality in the effect (a Semantic gender advantage). 
Fig. 3. Accuracy broken down by Gender Type and participant group (control group and PWA). The error bars indicate SE. The effect of Group is not reported, even though it is significant.

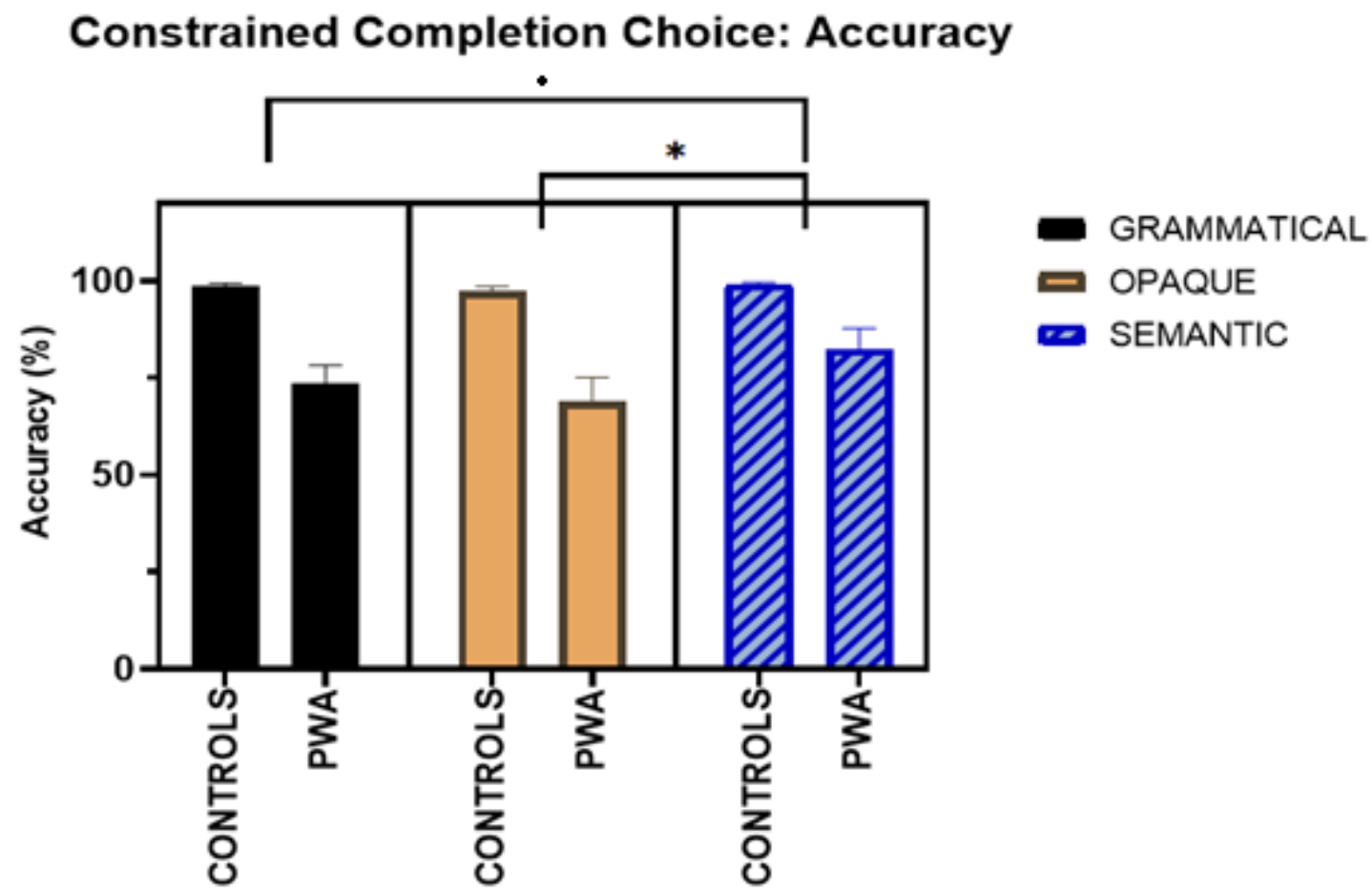


Fig. 4. Magnitude of the Semantic gender effect based on accuracy difference between the Semantic condition and the Grammatical condition as described in the main text. Individual PWA scores (stars where the individual performance significantly differed from the controls). More positive values indicate (proportionally) more accurate responses.

\section{Semantic gender effect (accuracy) Individual PWA scores}

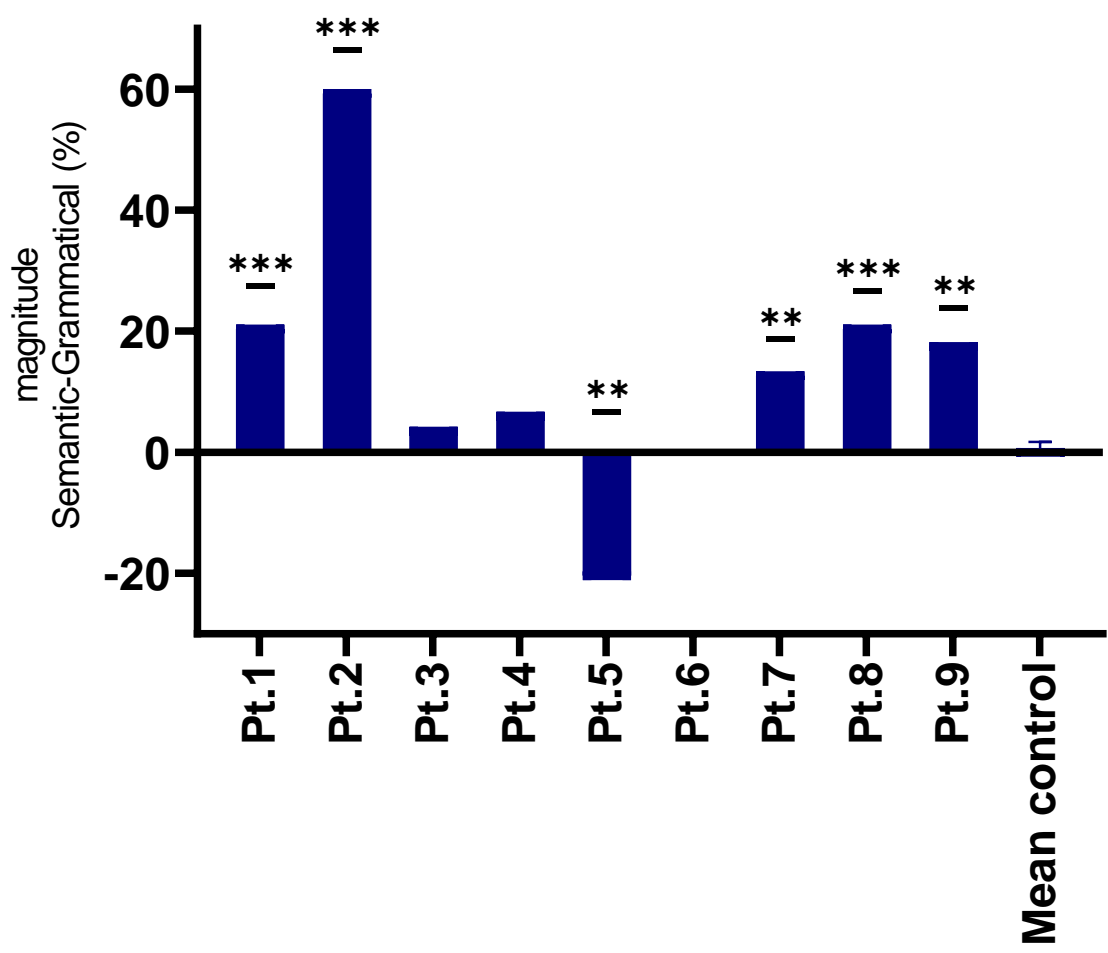

\section{Discussion}


We investigated whether grammatical and semantic gender are processed in different ways in sentence comprehension as proposed by the maximal input hypothesis (Barlow, 1993; Pollard and Sag, 1994; Vigliocco and Franck, 1999). We used experimental material similar to that in Vigliocco and Franck (1999), including a distractor with the opposite gender to that of the sentence subject, so as to induce a relatively high number of errors and avoid any possible ceiling effects. However, our experiment differed in two key respects from their study: we used a comprehension task and we tested PWA, a group that is more likely to show sharper differences in processing the two types of gender.

Our main finding was that semantic gender was processed faster and more accurately than grammatical gender (in both the Grammatical and Opaque conditions) by both the control group as well as PWA. This supports the hypothesis that gender type influences semantic comprehension. Crucially, we found a substantial difference in the magnitude of the Semantic gender effect in the PWA compared to the control group. The individual analysis highlighted that this effect was significantly larger for the majority of PWA than for controls. This finding further confirms our assumption that semantic gender provides salient information that supports both lexical processing and the computation of agreement. By contrast, although accuracy was above chance, grammatical gender agreement processing was impaired in PWA compared to controls (consistently with the cue-based retrieval model for sentence comprehension, Lewis and Van Dyke, 2003; Van Dyke and McElree, 2006; see also Parker et al.'s, 2017). The fact that the effect was of larger magnitude in PWA than controls supports our hypothesis that semantic gender helps PWA by making comprehension (and syntactic) processes easier; in contrast, computing grammatical gender requires greater cognitive resources. Below, we discuss this PWA Semantic gender effect in the context of our hypothesis and consider the role of cognitive control in sentence comprehension. 
Since semantic gender reflects the gender of its referent, it facilitates gender agreement tasks. This is because semantic information is retrieved during lexical access (see Friedmann et al., 2013) and strictly bound to meaning, two factors that facilitate lexical processing. We assume that semantic gender originates at the level of the conceptual representations that underpin lexical concepts, in line with studies that have demonstrated the existence of semantic priming (see Masson, 1995; Ortells et al., 2006). Importantly, in our task we found the two gender types were processed differently, suggesting that semantic gender could play an active role in lexical access. Compatible with cue-based retrieval models, which assume that cue weights (or salience) determine dependency retrieval (Lewis et al., 2006; Van Dyke and McElree, 2006; Parker et al., 2017), our results reveal that semantic gender is a higher-level feature than grammatical gender. The interference we observed was limited to (both) the grammatical gender conditions, which shared the same gender feature as sentence distractors. Conversely, semantic gender facilitated computation of agreement because more salient information was accessible during the retrieval process. For this reason, semantic gender was preserved in the memory of PWA, reducing the influence of grammatical gender candidates; by contrast, PWA's processing of both transparent and opaque grammatical gender was impaired. This cue-based retrieval model could also explain the lack of statistical difference between the Opaque and Grammatical conditions, since both have equally low salient features.

This result also confirms that the effect we observed was not driven by the degree of morphological transparency. If this had been the case, the Opaque condition would have been slower and less accurate. Caffarra et al., (2016) showed a difference between nouns with opaque and transparent grammatical gender, which we failed to observe in both groups. We assume this discrepancy is due to task differences: Caffarra et al.'s task entails explicit use of gender (i.e. gender categorization of bare nouns), while ours elicits procedural knowledge of gender in sentence comprehension. In fact, Gollan and Frost (2001) found that Hebrew bare 
nouns with irregular gender morphemes (which do not indicate noun gender) are processed less accurately and more slowly than bare transparent nouns, but this difference was eliminated these same nouns were computed together with the article to form a full noun phrase (procedural knowledge).

Impairment of inhibition and cognitive control (Faroqi-Shah et al., 2016) may play a role in the larger Semantic effect observed in PWA. In the context of sentence comprehension, PWA were overall able to process agreement at distance. However, their adjective choice mostly echoed the distractor's grammatical gender in the Grammatical and Opaque gender conditions, presumably because they were unable to inhibit this irrelevant but recent gender cue. This indicates that in PWA non-relevant information (the grammatical gender of the distractor) tends to reverberate and becomes more difficult to inhibit. Such findings are also in line with Lewis et al.'s (2006) computational principles of sentence comprehension, which describe how rapid retrieval of word is hindered by interference from similar items ('similarity-based interference', see also Van Dyke and Johns, 2012).

Alongside deficits in cognitive control, previous research showed that short-term and working memory explained poor performances in sentence comprehension depending on syntactic complexity and on the amount of semantic information available (Martin and Romani, 1994; Martin et al., 1994; Martin and He, 2004; Pettigrew and Hillis, 2014). Semantic and grammatical gender processing may entail two distinct paths one of which may be more taxing for WM and cognitive control. Processing agreement for grammatical gender relies more heavily on working memory (WM) - as Sagarra and Herschensohn's (2010) results suggest while the salient information in semantic gender facilitates lexical processing, probably requiring a smaller contribution from WM. PWA have weaker working memory than controls when processing sentences, and this may lead to the difficulties they display in the Grammatical and Opaque conditions relative to controls (see Minkina et al., 2018; Varkanitsa and Caplan, 
2018 for a discussion on short-term/working memory in aphasia). In fact, PWA were deceived by the distractor noun presented in the sentence and were apparently unable to disregard this distracting feature (i.e., the opposite gender). This irrelevant cue influenced their adjective choice for gender agreement. Despite the fact that this task put some demands on PWAs' WM that could have influenced their performance, we do not believe that WM overload was so complete as to have completely invalidated their results. If this had been the case, WM overload would have been reflected by drastic decreases in PWA's accuracy scores. They would have performed the task with such low accuracy that there would have been no differences observed between gender types.

In sum, we confirmed our hypothesis that semantic gender and grammatical gender are processed but incur different levels of difficulty for PWA and healthy participants. We also found the Semantic gender effect was of greater magnitude in most PWA than controls. This suggests that semantic gender is retained in this impaired population (regardless of aphasia type), whereas agreement for grammatical gender - of both transparent and opaque morphology - is partially disrupted. PWA's procedural knowledge of gender, although worse than that of controls, is generally spared. This may be the skill they employ during post-lexical processes when agreement is required (Cacciari and Cubelli, 2003, see also Roelofs et al., 1998). If so, it was the additional semantic feature exhibited by semantic gender that allowed PWA to perform similarly to the control group only in the semantic condition.

We had a heterogeneous group of PWA, and we did not measure agrammatism in production and comprehension. Nevertheless, we analysed individual PWA performances and the results demonstrated that the responses are unlikely to depend on aphasia type. In fact, Akhutina et al.'s (2001) work, which reported a gender priming effect in PWA (facilitation when prime and target were matched for gender), acknowledged that the gender priming effect did not correlate with any aphasia factor score, symptom, or classification. In addition, some 
research has been conducted on heterogeneous groups of PWA. Kulke and Blanken (2001), for example, grouped patients with various types of aphasia to study the preservation of grammatical gender. They discovered that in participants with Broca's and Wernicke's aphasia, the level of gender preservation was significantly above chance, with no significant difference between the two groups.

Moreover, the tasks we employed did not involve speaking or naming, which could have been more impaired in some participants than in others. Despite possible impairments, the PWA we analysed were able to complete the experiments and obtain a good accuracy score. It is known that a given brain lesion may produce a recognizable pattern of errors across different tasks (Whitworth et al., 2006). Together with neurological evidence, this supports the classification of aphasia types. Nevertheless, there is considerable variability across patients with similar lesions or types of aphasia. Two patients with the same lesion or diagnosis may not perform similarly, even on the same language tasks (Whitworth et al. 2006). Assigning PWA to particular types of aphasia cannot precisely predict linguistic performance. Consistently, Grunden et al. (2020) explored voluntary language switching in bilingual aphasia and demonstrated that PWA with the same aphasia diagnosis have varied and sometimes even opposite types of language control issues. We compared PWA's performance to non-braindamaged controls and showed that, in most cases, PWA performance was in line with that of the control participants (see Fig.2 and Fig.5). In addition, Pt.5, who uniquely produced the opposite pattern of accuracy to the other PWA, also showed a significant Semantic gender advantage in RT results. Conversely, the Semantic gender effect produced significantly slower RTs for Pt.1, who nevertheless clearly benefitted from higher accuracy in the same Semantic condition. We conclude that the presence of different aphasia types did not compromise our results. 
Moreover, we do not consider bilingualism to be a concern for our experiment because we found no substantial difference between bilinguals and monolinguals in the analysis of the subset of bilinguals. Note that both groups of participants had monolinguals and bilinguals and the gender of the stimuli was the same in both Spanish and Catalan. Future investigations should probe effects in bilinguals whose languages differ in the ascription of gender to check whether bilingualism is a confounding factor.

\section{Conclusion}

Semantic gender is assumed to be the first type of gender retrieved because this retrieval happens concurrently with lexical access at an early stage of processing. Semantic gender may also enhance word recognition in a sentence and - since it is bound to noun meaning - may induce longer-lasting effects than grammatical gender, which is retrieved later. This viewpoint is also supported by evidence from PWA, who were slower to inhibit irrelevant stimuli when processing agreement at distance. Further research using declarative knowledge of semantic gender in PWA will shed light on the nature of semantic gender processing.

\section{Acknowledgment}

MC was supported by the postdoctoral Ramón y Cajal fellowship (RYC-2013-14013), Agencia Estatal de Investigación (AEI, National Research Agency), and Fondo Europeo de Desarrollo Regional (FEDER, European Regional Development Fund) under project PSI201787784-R.

\section{Disclosure of interest}


The authors report no conflict of interest.

\section{References}

Akhutina, T., Kurgansky, A., Kurganskaya, M., Polinsky, M., Polonskaya, N., Larina, O., Bates, E., \& Appelbaum, M. (2001). Processing of Grammatical Gender in Normal and Aphasic Speakers of Russian. Cortex, 37(3), 295-326. https://doi.org/10.1016/S0010-9452(08)70576$\underline{8}$

Balota, D. A. (1992). Visual word recognition: The journey from feature to meaning. In M. Gernsbacher (ed.), Handbook of psycholinguistics, 303-357. New York: Academic Press. Barlow, D. H. (Ed.). (1993). Clinical handbook of psychological disorders: A step-by-step treatment manual, 2nd ed (pp. x, 534). The Guilford Press.

Bates, E. (1999). Language and the infant brain. Journal of Communication Disorders, 32(4), 195-205. https://doi.org/10.1016/S0021-9924(99)00015-5

Bates, E., Devescovi, A., Pizzamiglio, L., D'Amico, S., \& Hernandez, A. (1995). Gender and lexical access in Italian. Perception and Psychophysics, 57(6), 847-862. https://doi.org/10.3758/BF03206800

Bates, E., Marangolo, P., Pizzamiglio, L., \& Dick, F. (2001). Linguistic and Nonlinguistic Priming in Aphasia. Brain and Language, 76(1), 62-69. https://doi.org/10.1006/brln.2000.2391

Berg, T., \& Levelt, W. J. M. (1990). Speaking: From Intention to Articulation. The American Journal of Psychology, 103(3), 409. https://doi.org/10.2307/1423219

Bock, K., \& Levelt, W. J. M. (1994). Language Production: Grammatical Encoding. In M. A. Gernsbacher (Ed.), Handbook of Psycholinguistics. pp. 945-984. San Diego, CA: Academic Press.

Cacciari, C., \& Cubelli, R. (2003). The Neuropsychology of Grammatical Gender: An Introduction. Cortex, 39(3), 377-382. https://doi.org/10.1016/S0010-9452(08)70254-5 
Caffarra, S., Zimnukhova, S., \& Mancini, S. (2016). What usage can do: The effect of language dominance on simultaneous bilinguals' morphosyntactic processing. Linguistics Vanguard, 2(s1). https://doi.org/10.1515/lingvan-2016-0020

Chomsky, N. (1965). Aspects of the Theory of Syntax. Cambridge: MIT press.

Chomsky, N. (1981). Lectures on government and binding: the Pisa lectures. Holland: Foris Publications.

Corbett, G. G. (1991). Gender. Cambridge University Press. https://doi.org/10.1017/CBO9781139166119

Corbett, G. G. (2006). Agreement. Cambridge University press.

Crawford, J. R., \& Howell, D. C. (1998). Comparing an Individual's Test Score Against Norms Derived from Small Samples. The Clinical Neuropsychologist, 12(4), 482-486. https://doi.org/10.1076/clin.12.4.482.7241

Damian, M. F. (2010). Does variability in human performance outweigh imprecision in response devices such as computer keyboards? Behavior Research Methods, 42(1), 205-211. https://doi.org/10.3758/BRM.42.1.205

Davis, C. J., \& Perea, M. (2005). BuscaPalabras: A program for deriving orthographic and phonological neighborhood statistics and other psycholinguistic indices in Spanish. Behavior Research Methods, 37(4), 665-671. https://doi.org/10.3758/bf03192738

Faroqi-Shah, Y., Sampson, M., Pranger, M., \& Baughman, S. (2016). Cognitive control, word retrieval and bilingual aphasia: Is there a relationship? Journal of Neurolinguistics. https://doi.org/10.1016/j.jneuroling.2016.07.001

Forster, K. I., \& Forster, J. C. (2003). DMDX: A Windows display program with millisecond accuracy. Behavior Research Methods, Instruments, \& Computers, 35(1), 116-124. https://doi.org/10.3758/BF03195503 
Franzon, F., Peressotti F., Arcara G., \& Semenza C. (2013). Gender Agreement: a comparate psycholinguistic and aphasia case study. Academy of Aphasia-52nd Annual Meeting. Frontiers in Psychology, 5-7. https://doi.org/conf.fpsyg.2014.64.00056

Friedmann N., Biran, M., \& Dotan, D. (2013). Lexical retrieval and its breakdown in aphasia and developmental language impairment. In Boeckx, C., \& Grohmann, K.K. (Eds.), The Cambridge Handbook of Biolinguistics. Cambridge: Cambridge University Press. https://doi.org/doi:10.1017/CBO9780511980435.021

Friedmann, N., \& Biran, M. (2003). When is Gender Accessed? A Study of Paraphasias in Hebrew Anomia. Cortex, 39(3), 441-463. https://doi.org/10.1016/S0010-9452(08)70258-2

Friedmann, N., Biran, M., \& Dotan, D. (2013). Lexical retrieval and its breakdown in aphasia and developmental language impairment. In C. Boeckx \& K. K. Grohmann (Eds.), The Cambridge Handbook of Biolinguistics (pp. 350-374). Cambridge University Press. https://doi.org/10.1017/CBO9780511980435.021

Gollan, T. H., \& Frost, R. (2001). Two routes to grammatical gender: Evidence from Hebrew. Journal of Psycholinguistic Research, 30(6), 627-651. https://doi.org/10.1023/A:1014235223566

Goodglass, H. (2000). Grammatical gender is not always syntactic. In Grodzinsky, Y., Shapiro, L.P., \& Swinney, D. (Eds), Language and the brain: representation and processing. San Diego: Academic Press. https://doi.org./10.1016/B978-012304260-6/50015-3

Grunden, N., Piazza, G., García-Sánchez, C., \& Calabria, M. (2020). Voluntary Language Switching in the Context of Bilingual Aphasia. Behavioral Sciences, 10(9), 141. https://doi.org/10.3390/bs10090141

Kempen, G., \& Hoenkamp, E. (1987). An Incremental Procedural Grammar for Sentence Formulation. Cognitive Science, 11(2), 201-258. https://doi.org/10.1207/s15516709cog1102_5 
Kertesz, A. (1982). The Western Aphasia Battery: Test Manual, Stimulus Cards, Test Booklets (Test Kit). New York: Grune and Stratton. https://doi.org/10.1080/01688638508401277 Kertesz, A., Pascual, Á. P.-L., \& García, A. P.-L. (1990). Batería de afasías “Western”: : (The western aphasia battery en versión y adaptación castellana). Manual del test. Nau Llibres. https://dialnet.unirioja.es/servlet/libro?codigo $=90650$

Kulke, F., \& Blanken, G. (2001). Phonological and syntactic influences on semantic misnamings in aphasia. Aphasiology, 15(1), 3-15. https://doi.org/10.1080/02687040042000070

Levelt, W. J. M. (1989). Speaking: From Intention to Articulation. Cambridge, MA: MIT Press. https://doi.org/10.2307/1423219

Lewis, R. L., Vasishth, S., \& Van Dyke, J. A. (2006). Computational principles of working memory in sentence comprehension. Trends in Cognitive Sciences, 10(10), 447-454. https://doi.org/10.1016/j.tics.2006.08.007

MacDonald, M. C., Pearlmutter, N. J., \& Seidenberg, M. S. (1994). The lexical nature of syntactic ambiguity resolution. Psychological Review, 101(4), 676-703. https://doi.org/10.1037/0033$\underline{295 X .101 .4 .676}$

Manenti, R., Repetto, C., Bentrovato, S., Marcone, A., Bates, E., \& Cappa, S.F. (2004). The effects of ageing and Alzheimer's disease on semantic and gender priming. Brain, 127(10), 2299-2306. https://doi.org/10.1093/brain/awh264

Martin, R.C., \& He, T. (2004). Semantic short-term memory and its role in sentence processing: A replication. Brain and Language, 89(1), 76-82. https://doi.org/10.1016/S0093$\underline{934 X(03) 00300-6}$

Martin, R.C., \& Romani, C. (1994). Verbal working memory and sentence comprehension: A multiple-components view. Neuropsychology, 8(4), 506-523. https://doi.org/10.1037/0894$\underline{4105.8 .4 .506}$ 
Martin, R.C., Shelton, J. R., \& Yaffee, L. S. (1994). Language Processing and Working Memory: Neuropsychological Evidence for Separate Phonological and Semantic Capacities. Journal of Memory and Language, 33(1), 83-111. https://doi.org/10.1006/jmla.1994.1005

Masson, M. E. J. (1995). A distributed memory model of semantic priming. Journal of Experimental Psychology: Learning, Memory, and Cognition, 21(1), 3-23. https://doi.org/10.1037/0278-7393.21.1.3

Miceli, G., Turriziani, P., Caltagirone, C., Cappaso, R., Tomaiuolo, F., Caramazza, A. (2002). The neural correlates of grammatical gender: An fMRI investigation. Journal of Cognitive Neuroscience, 14: 618-628, 2002. https://doi.org/10.1162/08989290260045855

Minkina, I., Salis, C., \& Martin, N. (2018). Short-term and working memory deficits in aphasia: Current issues in theory, evidence, and treatment. Journal of Neurolinguistics, 48, 1-3. https://doi.org/10.1016/j.jneuroling.2018.07.001

Mondini, S., Luzzatti, C., \& Semenza, C. (1999). Grammatical gender knowledge in an Italian agrammatic patient. Brain and Language, 69(3), 278-281.

Ortells, J. J., Vellido, C., Daza, M. T., \& Noguera, C. (2006). Efectos de priming semántico con y sin conciencia perceptiva. [Semantic priming effects with and without perceptual awareness.]. Psicológica, 27(2), 225-242.

Parker, D., Shvartsman, M., \& Van Dyke, J. A. (2017). The cue-based retrieval theory of sentence comprehension: New findings and new challenges. In L. Escobar, V. Torrens, \& T. Parodi (Eds.) Language Processing and Disorders (pp. 121-144). Newcastle: Cambridge Scholars Publishing.

Pettigrew, C. \& Hillis, A. E. (2014) Role for memory capacity in sentence comprehension: Evidence from acute stroke. Aphasiology, 28:10, 1258-1280, https://doi.org/10.1080/02687038.2014.919436 
Pollard, C., \& Sag, I. A. (1994). Head-Driven Phrase Structure Grammar. Chicago: The University of Chicago Press.

Repetto, C., Manenti R., Cappa S.F., Miniussi C., \& Riva G. (2009). Semantic and gender priming in frontotemporal dementia. Annual Review of Cybertherapy and Telemedicine, 237-239. https://doi.org/10.3233/978-1-60750-017-9-237

Roelofs, A., Meyer, A.S., \& Levelt, W. J. M. (1998). A case for the lemma/lexeme distinction in models of speaking: Comment on Caramazza and Miozzo (1997). Cognition, 69, 219-230. https://doi.org/10.1016/s0010-0277(98)00056-0

Sagarra, N., \& Herschensohn, J. (2010) The role of proficiency and working memory in gender and number agreement processing in L1 and L2 Spanish. Lingua, 120(8), 2022-2039. https://doi.org/10.1016/j.lingua.2010.02.004

Scarnà, A., \& Ellis, A. W. (2002). On the assessment of grammatical gender knowledge in aphasia: The danger of relying on explicit, metalinguistic tasks. Language and Cognitive Processes, 17(2), 185-201. https://doi.org/10.1080/0169096014300038

Schiller, N. O., Munte, T. F., Horemans, I., \& Jansma, B. M. (2003). The influence of semantic and phonological factors on syntactic decisions: An event-related brain potential study. Psychophysiology, 40, 869-869. https://doi.org/10.1111/1469-8986.00105

Schiller, N. O., Schuhmann, T., Neyndorff, A. C., \& Jansma, B. M. (2006). The influence of semantic category membership on syntactic decisions: A study using event-related brain potentials. Brain Research, 1082(1), 153-164. https://doi.org/10.1016/j.brainres.2006.01.087

Semenza, C., \& Luzzatti, C. (2019) Disturbi lessicali nell'afasia. In Denes et al. (Eds), Manuale di Neuropsicologia, Zanichelli, Bologna.

Van Dyke, J. A. (2007). Interference effects from grammatically unavailable constituents during sentence processing. Journal of Experimental Psychology: Learning, Memory, and Cognition, 33(2), 407-430. https://doi.org/10.1037/0278-7393.33.2.407 
Van Dyke, J. A., \& Johns, C. L. (2012). Memory Interference as a Determinant of Language Comprehension: Interference in Comprehension. Language and Linguistics Compass, 6(4), 193-211. https://doi.org/10.1002/lnc3.330

Van Dyke, J. A., \& McElree, B. (2006). Retrieval interference in sentence comprehension. Journal of Memory and Language, 55(2), 157-166. https://doi.org/10.1016/j.jml.2006.03.007 Varkanitsa, M., \& Caplan, D. (2018). On the association between memory capacity and sentence comprehension: Insights from a systematic review and meta-analysis of the aphasia literature. Journal of Neurolinguistics, 48, 4-25. https://doi.org./10.1016/j.jneuroling.2018.03.003

Vigliocco, G., Butterworth, B., \& Semenza, C. (1995). Constructing Subject-Verb Agreement in Speech: The Role of Semantic and Morphological Factors. Journal of Memory and Language, 34(2), 186-215. https://doi.org/10.1006/jmla.1995.1009

Vigliocco, Gabriella, \& Franck, J. (1999). When Sex and Syntax Go Hand in Hand: Gender Agreement in Language Production. Journal of Memory and Language, 40(4), 455-478. https://doi.org/10.1006/jmla.1998.2624

Vigliocco, Gabriella, Butterworth, B., \& Garrett, M. F. (1996). Subject-verb agreement in Spanish and English: Differences in the role of conceptual constraints. Cognition, 61(3), 261-298. https://doi.org/10.1016/S0010-0277(96)00713-5

Whitworth, A, Webster J, \& Howard D. (2006). A Cognitive neuropsychological approach to assessment and intervention in aphasia. Hove and New York: Psychology Press. https://doi.org/10.4324/9781315852447 


\section{Footnote}

${ }^{1}$ Throughout this paper we will refer to the gender of the referent and biological sex as the source for semantic gender. Without doubt, gender and sex have two substantially different meanings. Nevertheless, for the purpose of this paper, it is not always easy to determine which is the more appropriate term, because semantic gender pertains to both animals and humans. When referring to the semantic gender of human nouns, it is probably more correct to say it originates from gender, although (but this is not the aim of the paper) we do not know how semantic gender would be conceptualized in the case of non-correspondence between gender identity and sex. 
Table 1. Individual scores for the Western Aphasia Battery

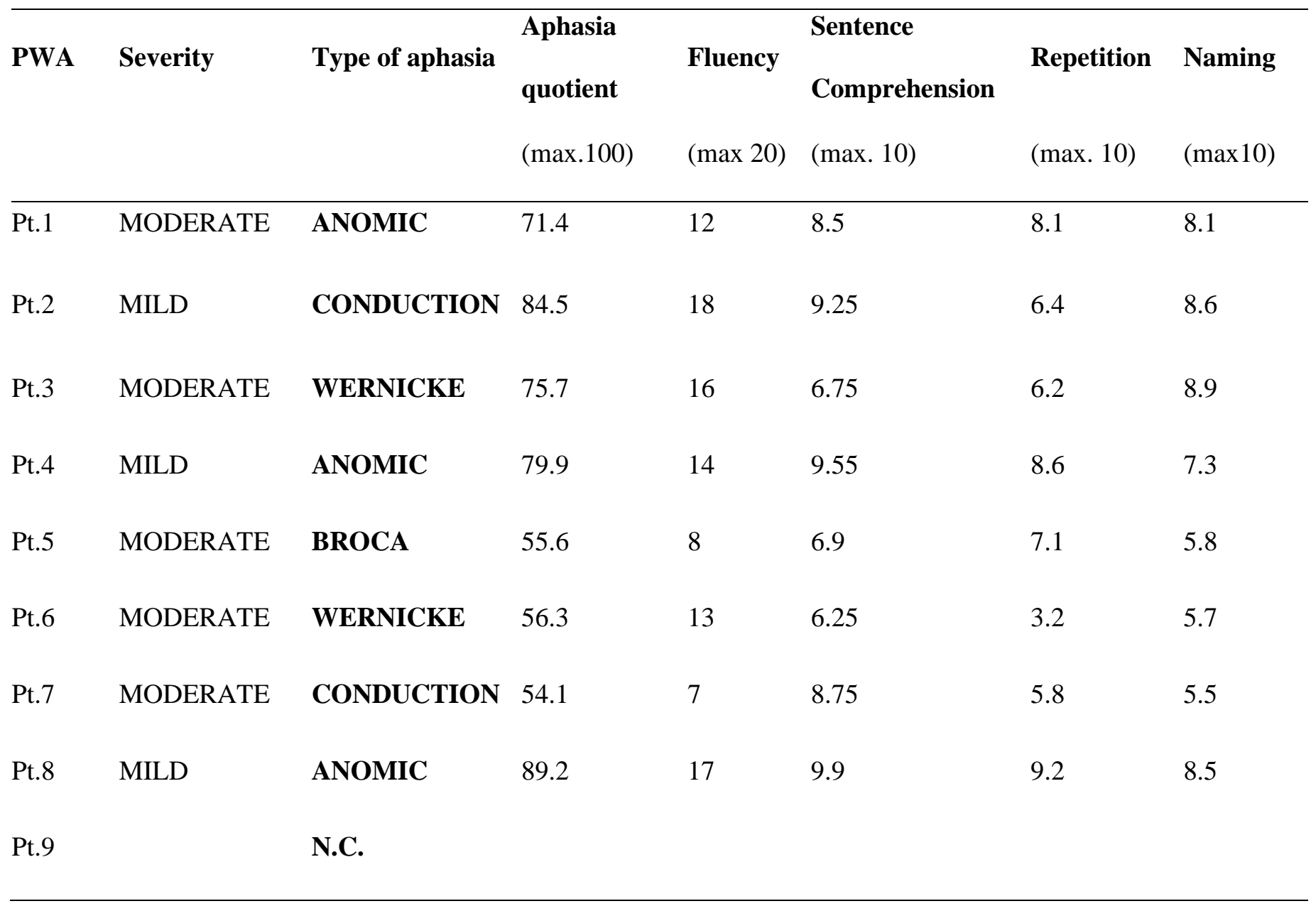

APPENDIX A. Examples of sentences predicative and adjectives.

El esposo en la iglesia está Semantic condition

The spouse in the church is

El mono en la clínica está

The monkey in the clinic is
EMOCIONADA EMOCIONADO

EXCITED

NERVIOSO

NERVIOSA

NERVOUS 


\begin{tabular}{llll}
\hline La comida en el plato está & Grammatical condition & SABROSA & SABROSO \\
The food on the dish is & & TASTY & \\
\hline La fuente del suelo está & Opaque condition & SECA & SECO \\
The fount on the ground is & & DRY & \\
& & & \\
\end{tabular}

